\title{
Gevrey Estimates of Formal Solutions for Certain Moment Partial Differential Equations with Variable Coefficients
}

\author{
Maria Suwińska ${ }^{1}$ \\ Received: 7 February 2020 / Published online: 21 July 2020 \\ (C) The Author(s) 2020
}

\begin{abstract}
The goal of this paper is to investigate Gevrey properties of formal solutions of certain generalized linear partial differential equations with variable coefficients. In particular, we extend the notion of moment partial differential equations to include differential operators that are not connected with kernel functions. Using the modified version of Nagumo norms and the properties of the Newton polygon we compute the Gevrey estimate for formal solutions of such generalized partial differential equations.
\end{abstract}

Keywords Formal solutions - Generalized partial differential equations · Nagumo norms · Newton polygon $\cdot$ Gevrey order

Mathematics Subject Classification (2010) 35C10 · 35G10

\section{Introduction}

Over the last decade many studies have been devoted to the topic of the Gevrey properties of formal series solutions of linear partial differential equations. Main topics of interest include inhomogeneous PDEs with constant coefficients [3], the heat equation and its various generalizations $[2,8,9]$ and PDEs with time-dependent coefficients [10]. Those results have also been generalized to the case of moment partial differential equations with constant and time-dependent coefficients [5-7].

In this paper we aim to further generalize the notion of moment partial differential equations. To this end, we define Gevrey-type sequences and operators, which maintain many of the characteristics of the moment functions and moment differential operators, respectively. More details on the subject are given in Section 2.

Maria Suwińska

m.suwinska@op.pl

1 Faculty of Mathematics and Natural Sciences, College of Science, Cardinal Stefan Wyszyński University, Wóycickiego 1/3, 01-938 Warsaw, Poland 
We focus our attention on a general Cauchy problem of the form:

$$
\left\{\begin{array}{rl}
P\left(\partial_{m_{0}, t}, \partial_{m_{1}, z_{1}}, \ldots, \partial_{m_{N}, z_{N}}\right) u(t, \mathbf{z}) & =f(t, \mathbf{z}) \\
\partial_{m_{0}, t}^{j} u(0, \mathbf{z}) & =\phi_{j}(\mathbf{z}) \text { for } 0 \leq j<M
\end{array},\right.
$$

where $P\left(\partial_{m_{0}, t}, \partial_{m_{1}, z_{1}}, \ldots, \partial_{m_{N}, z_{N}}\right)$ is a linear operator with coefficients depending on both variables $t \in \mathbb{C}$ and $\mathbf{z} \in \mathbb{C}^{N}$, and $\partial_{m_{0}, t}, \partial_{m_{1}, z_{1}}, \ldots, \partial_{m_{N}, z_{N}}$ are Gevrey-type differential operators (see Definition 3). Our goal is to connect the Gevrey order of its solution with the orders of the inhomogeneity $f$ and the variable coefficients.

While analyzing an equation very similar to the ones studied in [7, 10], we mostly draw inspiration from $[2,8]$, where the concept of Nagumo norms is utilized. Below we recall the definition used in papers mentioned above:

Definition 1 Let us consider a function $f$ holomorphic on a disc $D_{R}=\{z \in \mathbb{C}:|z|<R\}$, $p \geq 0$ and $0<r<R$. Then the Nagumo norm $\|f\|_{p, r}$ of $f$ is defined by

$$
\|f\|_{p, r}=\sup _{z \in D_{r}}\left|f(z)(r-|z|)^{p}\right| .
$$

The usefulness of this family of norms lays in their properties. Namely, for any two functions $f_{1}, f_{2}$ holomorphic on $D_{R}$, non-negative numbers $p, q$ and $0<r<R$ we have:

- $\quad\left\|f_{1} f_{2}\right\|_{p+q, r} \leq\left\|f_{1}\right\|_{p, r}\left\|f_{2}\right\|_{q, r}$,

- $\quad\left\|\partial_{z} f_{1}\right\|_{p+1, r} \leq C(p+1)\left\|f_{1}\right\|_{p, r}$ for a certain constant $C>0$,

- $\left|f_{1}(z)\right| \leq\left\|f_{1}\right\|_{p, r}(r-|z|)^{-p}$ for all $z \in D_{r}$.

Especially the second attribute is very important, because it enables us to estimate $\partial_{z} f$ by $f$ without making shrinking of the domain necessary. Unfortunately, the standard definition mentioned above could not be used to determine the Gevrey order of the solution of (1). This fact made creating a new tool with similar properties necessary and so certain modifications were utilized to generalize the Nagumo norms in a way more fitted to our needs. Indeed, it has been proven in Lemmas 2, 4 and 7 that the properties listed above hold for the modified Nagumo norms defined in Section 2. Moreover, in some cases both norms coincide for $z \in \mathbb{C}$.

The main result of the paper, Theorem 1, shows that under certain additional assumptions the Gevrey order of the formal solution of (1) is equal to the reverse of the slope of the first non-horizontal segment in the Newton polygon for $P$. This outcome is a direct generalization of results found in $[2,7,8,10]$.

The paper is structured as follows. In Section 2 we first establish the notation and then move on to defining Gevrey-type sequences and operators and presenting their characteristics. Some of the most important examples are listed there as well. We also recall the definition of the Newton polygon and alter it to better accommodate the structure of (1). Section 3 is entirely devoted to the modified Nagumo norms. Several important properties of these norms are presented. We also go into further detail on their connection with the Gevrey order of a function. Section 4 contains all conditions, under which we would like to consider our initial equation. After that we present the proof of Proposition 2, from which the main result of this paper follows. Ideas for further study are included in Section 5. 


\section{Preliminaries}

\subsection{Notation}

Let us introduce the notation that will be used throughout this paper. First, note that by $\mathbb{N}_{0}$ we shall denote the set of all non-negative integers and $\mathbb{N}=\mathbb{N}_{0} \backslash\{0\}$.

For any positive integer $N$, by $D_{R}^{N}$ we denote a polydisc with a center at 0 and a radius $R>0$, i.e., a set

$$
D_{R}^{N}=\left\{\mathbf{z}=\left(z_{1}, \ldots, z_{N}\right) \in \mathbb{C}^{N}:\left|z_{j}\right|<R \text { for } j=1, \ldots, N\right\} .
$$

If $\Omega \subset \mathbb{C}^{N}$ and a function $f$ is holomorphic on the set $\Omega$ then we write $f \in \mathscr{O}(\Omega)$.

For any function $f \in \mathscr{O}\left(D_{R}\right)$ by $\operatorname{ord}_{t}(f)$ we denote the order of zero of the function $f$ at $t=0$.

A space of all formal power series $\hat{u}(t)=\sum_{n=0}^{\infty} u_{n} t^{n}$ with coefficients from a given Banach space $\mathbb{E}$ will be denoted by $\mathbb{E}[[t]]$. Throughout this paper we will restrict ourselves to the case when $\mathbb{E}$ is the space $\mathscr{O}\left(D_{R}^{N}\right) \cap C\left(\bar{D}_{R}^{N}\right)$ of all functions holomorphic on a polydisc $D_{R}^{N} \subset \mathbb{C}^{N}$ and continuous on its closure, equipped with the standard norm $\|f\|=\sup _{\zeta \in D_{R}^{N}}|f(\zeta)|$.

For all multi-indices $\alpha=\left(\alpha_{1}, \alpha_{2}, \ldots, \alpha_{N}\right), \beta=\left(\beta_{1}, \beta_{2}, \ldots, \beta_{N}\right)$ in $\mathbb{N}_{0}^{N}$, points $\mathbf{z}=$ $\left(z_{1}, z_{2}, \ldots, z_{N}\right) \in \mathbb{C}^{N}$ and $\mathbf{s}=\left(s_{1}, s_{2}, \ldots, s_{N}\right) \in \mathbb{R}^{N}$, and $A \in \mathbb{R}$ the following notation will be used:

$$
\begin{array}{rl}
\alpha+\beta=\left(\alpha_{1}+\beta_{1}, \alpha_{2}+\beta_{2}, \ldots, \alpha_{N}+\beta_{N}\right) & \alpha \cdot \beta=\sum_{j=1}^{N} \alpha_{j} \beta_{j} \\
\alpha \leq \beta \Longleftrightarrow \alpha_{j} \leq \beta_{j} \text { for } j=1, \ldots, N & |\alpha|=\sum_{j=1}^{N} \alpha_{j} \\
\mathbf{z}^{\mathbf{s}}=z_{1}^{s_{1}} z_{2}^{s_{2}} \ldots z_{N}^{s_{N}} & A \alpha=\left(A \alpha_{1}, A \alpha_{2}, \ldots, A \alpha_{N}\right) \\
\mathbf{0}=(0,0, \ldots, 0) . &
\end{array}
$$

Let $a(\mathbf{z})=\sum_{\gamma \in \mathbb{N}_{0}^{N}} a_{\gamma} \mathbf{z}^{\gamma}$ and $b(\mathbf{z})=\sum_{\gamma \in \mathbb{N}_{0}^{N}} b_{\gamma} \mathbf{z}^{\gamma}$ be two formal power series. Then we call $b(\mathbf{z})$ a majorant of $a(\mathbf{z})$ and write $a(\mathbf{z}) \ll b(\mathbf{z})$ if $\left|a_{\gamma}\right| \leq b_{\gamma}$ for every $\gamma \in \mathbb{N}_{0}^{N}$.

\subsection{Gevrey-Type Sequences and Operators}

Definition 2 Let $m(n)$ denote a sequence of positive numbers such that $m(0)=1$. Then $m(n)$ will be called a Gevrey-type sequence of order $s \geq 0$ if there exist constants $a, A>0$ satisfying the condition

$$
a^{n} n !^{s} \leq m(n) \leq A^{n} n !^{s} \text { for any } n \in \mathbb{N}_{0} .
$$

Note that Definition 2 encompasses a wide variety of sequences. In particular, (2) describes one of the main characteristics of so-called moment functions, which are in turn directly connected to kernel functions. For more information on the topic, we refer the reader to [1, Section 5.5]. In this paper we depart from this concept, but many elements used in the theory of moment functions and moment differential operators can be easily adapted to this more general family of sequences. We devote the next part of this chapter to presenting their various properties. 
Proposition 1 (see [1, Theorems 31 and 32]) Let $m_{1}$ and $m_{2}$ be two Gevrey-type sequences of non-negative orders $s_{1}$ and $s_{2}$, respectively. Then $m_{1} \cdot m_{2}$ is a Gevrey-type sequence of order $s_{1}+s_{2}$. Moreover, if $s_{1}>s_{2}$ then $\frac{m_{1}}{m_{2}}$ is a Gevrey-type sequence of order $s_{1}-s_{2}$.

Below we present several examples of Gevrey-type sequences.

\section{Example 1}

- Function $\Gamma_{s}(n)=\Gamma(1+s n)$, where $s>0$, is a Gevrey-type sequence of order $s$.

- Let us put $[n]_{q}=1+q+\ldots+q^{n-1}=\frac{q^{n}-1}{q-1}$ for a fixed $q \in(0,1)$. Then a sequence given by the formula

$$
[n]_{q} != \begin{cases}{[n]_{q} \cdot[n-1]_{q} \cdot \ldots \cdot[1]_{q}} & \text { for } n \geq 1 \\ 1 & \text { for } n=0\end{cases}
$$

is a Gevrey-type sequence of order 0 . Indeed, it is easy to see that

$$
1 \leq[n]_{q} ! \leq \frac{1}{(1-q)^{n}} \text { for any } n \in \mathbb{N}_{0} .
$$

We will also use a special class of sequences defined below.

Definition 3 If $m(n)$ is a sequence of positive numbers such that $m(0)=1$ and $s \geq 0$ then we shall call $m(n)$ a regular Gevrey-type sequence of order $s$ if there exist constants $a, A>0$ satisfying the condition

$$
a(n+1)^{s} \leq \frac{m(n+1)}{m(n)} \leq A(n+1)^{s} \text { for any } n \in \mathbb{N}_{0} .
$$

Remark 1 It is easy to observe that every regular Gevrey-type sequence of order $s$ is a Gevrey-type sequence of the same order and for the same constants $a$ and $A$.

Regular Gevrey-type sequences satisfy the following properties:

Lemma 1 (compare [7, Lemma 2.1])

(i) The class of regular Gevrey-type sequences is closed under multiplication and division.

(ii) The class of regular Gevrey-type sequences contains the sequence $\Gamma_{s}(n)=\Gamma(1+s n)$.

Definition 4 (see [3]) Let $m$ be a Gevrey-type sequence. Then we define a Gevrey-type differential operator $\partial_{m, t}: \mathbb{E}[[t]] \rightarrow \mathbb{E}[[t]]$ by the formula:

$$
\partial_{m, t}\left(\sum_{n=0}^{\infty} \frac{u_{n}}{m(n)} t^{n}\right):=\sum_{n=0}^{\infty} \frac{u_{n+1}}{m(n)} t^{n} .
$$

A couple of examples of Gevrey-type differential operators can be found below:

\section{Example 2}

- For $m(n)=n$ ! operator $\partial_{m, t}$ coincides with the standard derivative $\partial_{t}$.

- Consider a Gevrey-type sequence $\Gamma_{s}(n)$ defined in Example 1. Then we have

$$
\partial_{t}^{s} u\left(t^{s}\right)=\left(\partial_{\Gamma_{s}, w} u\right)\left(t^{s}\right)
$$


with $\partial_{t}^{s}$ being the Caputo fractional derivative.

- Suppose that $m(n)=[n]_{q}$ ! for a certain $q \in(0,1)$. We have established in Example 1 that $m(n)$ is a Gevrey-type sequence of order 0 . We will show that the $q$-difference differential operator given by

$$
D_{q, t} u(t)=\frac{u(q t)-u(t)}{q t-t}
$$

is equal to the Gevrey-type operator $\partial_{m, t}$ for any function $u(t)=\sum_{n=0}^{\infty} \frac{u_{n}}{[n]_{q} !} t^{n}$. Indeed, let us note that

$$
D_{q, t} t^{n}=\frac{q^{n} t^{n}-t^{n}}{q t-t}=[n]_{q} t^{n-1} \text { for any } n \in \mathbb{N} \text {. }
$$

Hence,

$$
D_{q, t} u(t)=\sum_{n=1}^{\infty} \frac{u_{n}}{[n]_{q} !}[n]_{q} t^{n-1}=\sum_{n=1}^{\infty} \frac{u_{n}}{[n-1]_{q} !} t^{n-1}=\partial_{m, t}\left(\sum_{n=0}^{\infty} \frac{u_{n}}{[n]_{q} !} t^{n}\right) .
$$

We can now recall the basic definition of the Gevrey order.

Definition 5 The formal series $\hat{u}(t)=\sum_{n=0}^{\infty} u_{n} t^{n} \in \mathbb{E}[[t]]$ is of Gevrey order $s$ if and only if there exist $B, C>0$ such that

$$
\left\|u_{n}\right\|_{\mathbb{E}} \leq B C^{n} n !^{s} \quad \text { for } \quad n \in \mathbb{N}_{0} .
$$

Then we write that $\hat{u}(t) \in \mathbb{E}[[t]]_{s}$.

Remark 2 Definition 5 implies that any formal power series of Gevrey order 0 is convergent.

\subsection{The Newton Polygon}

The Newton polygon for linear partial differential operators with variable coefficients has been introduced in [11]. The notion was later extended in [6] to moment partial differential equations with constant coefficients with two variables. The definition presented below is based on [7], where the Newton polygon was defined for linear moment partial differential operators with time-dependent coefficients.

Let $m_{0}, m_{1}, \ldots, m_{N}$ be Gevrey-type sequences of positive orders $s_{0}, s_{1}, \ldots, s_{N}$, respectively, and suppose that $\partial_{m_{0}, t}, \partial_{m_{1}, z_{1}}, \ldots, \partial_{m_{N}, z_{N}}$ are Gevrey-type differential operators. For $\mathbf{m}=\left(m_{1}, m_{2}, \ldots, m_{N}\right)$ and any multi-index $\alpha \in \mathbb{N}_{0}^{N}$ we use a notation $\partial_{\mathbf{m}, \mathbf{z}}^{\alpha}:=$ $\partial_{m_{1}, z_{1}}^{\alpha_{1}} \partial_{m_{2}, z_{2}}^{\alpha_{2}} \ldots \partial_{m_{N}, z_{N}}^{\alpha_{N}}$. Suppose that $J \subset \mathbb{N}_{0}$ and $A \subset \mathbb{N}_{0}^{N}$ are finite sets of indices. We shall consider an operator of the form:

$$
P\left(t, \mathbf{z}, \partial_{m_{0}, t}, \partial_{\mathbf{m}, \mathbf{z}}\right)=\sum_{(j, \alpha) \in J \times A} a_{j, \alpha}(t, \mathbf{z}) \partial_{m_{0}, t}^{j} \partial_{\mathbf{m}, \mathbf{z}}^{\alpha},
$$

where $a_{j, \alpha}(t, \mathbf{z})$ are holomorphic with respect to $t$ in an open neighborhood of zero for every $(j, \alpha) \in J \times A$. Furthermore let $\mathbf{s}=\left(s_{1}, \ldots, s_{N}\right)$.

Definition 6 We define the Newton polygon for the operator $P$ given by (4) as

$$
N\left(P, s_{0}, \mathbf{s}\right)=\operatorname{conv}\left\{\Delta\left(s_{0} j+\mathbf{s} \cdot \alpha, \operatorname{ord}_{t}\left(a_{j, \alpha}\right)-j\right):(j, \alpha) \in J \times A, a_{j, \alpha} \not \equiv 0\right\},
$$

where $\Delta(a, b)=\left\{(x, y) \in \mathbb{R}^{2}: x \leq a, y \geq b\right\}$ for any $a, b \in \mathbb{R}^{2}$. 
An example of a Newton polygon can be seen on Fig. 1.

\section{Modified Nagumo Norms}

Definition 7 For any $a \in \mathbb{N}_{0}$ and $s \geq 0$ let us define a power series $\Theta_{s}^{(a)}(x)$ by the formula

$$
\Theta_{s}^{(a)}(x):=\sum_{n=0}^{\infty} \frac{(n+a) !^{s}}{n !^{s}} x^{n} .
$$

Using series $\Theta_{s}^{(a)}(x)$ we can define a modified version of the Nagumo norm.

Definition 8 For any $\mathbf{s} \in[1, \infty)^{N}, \alpha \in \mathbb{N}^{N}, 0<r<R$ and $\mathbf{z} \in D_{r}^{N}$ we define the modified Nagumo norm $\|f(\mathbf{z})\|_{\alpha, r, \mathbf{s}}$ of $f \in \mathscr{O}\left(D_{R}^{N}\right)$ by the formula:

$$
\|f(\mathbf{z})\|_{\alpha, r, \mathbf{s}}:=\inf \left\{A \geq 0: f(\mathbf{z}) \ll A \prod_{i=1}^{N} \frac{1}{r^{\alpha_{i}}\left(\alpha_{i}-1\right) ! s_{i}} \Theta_{s_{i}}^{\left(\alpha_{i}-1\right)}\left(\frac{z_{i}}{r}\right)\right\} .
$$

It is worth mentioning that for $N=1$ and $s=1$ Definition 8 coincides with the standard way of defining the Nagumo norm. Indeed, let us take $q \in \mathbb{N}$ and $0<r<R$. Then, for any function $f$, (6) takes form

$$
\|f(z)\|_{q, r, 1}:=\inf \left\{A \geq 0: f(z) \ll A \frac{1}{r^{q}(q-1) !} \Theta_{1}^{(q-1)}\left(\frac{z}{r}\right)\right\} .
$$

Note that

$$
\Theta_{1}^{(q-1)}\left(\frac{z}{r}\right)=\sum_{n=0}^{\infty} \frac{(n+q-1) !}{n !} \frac{z^{n}}{r^{n}}=\left.\frac{\mathrm{d}^{q-1}}{\mathrm{~d} u^{q-1}}\left(\sum_{n=0}^{\infty} u^{n}\right)\right|_{u=r^{-1} z},
$$

which gives us

$$
\sup _{|z|<\rho}|f(z)| \leq \frac{A}{r^{q}(q-1) !} \frac{r^{q}(q-1) !}{(r-|z|)^{q}}=\frac{A}{(r-|z|)^{q}}
$$

for any $\rho<r$. Hence, $A \geq(r-\rho)^{q} \sup _{|z|<\rho}|f(z)|$.

Remark 3 Note that $\|\cdot\|_{\alpha, r, \mathbf{s}}$ is a norm on $\mathscr{O}\left(D_{R}^{N}\right)$ for any $\alpha \in \mathbb{N}^{N}, 0<r<R$ and $\mathbf{s} \in[1, \infty)^{N}$.

Fig. 1 An example of a Newton polygon

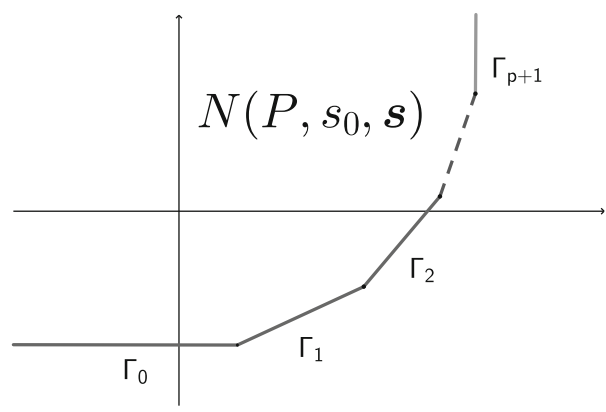


Remark 4 For the multi-index $\mathbf{0}$ we put $\|f(\mathbf{z})\|_{\mathbf{0}, r, \mathbf{s}}$ equal to the standard norm in the space $\ell_{1}$ for any $0<r<R$ and $\mathbf{s} \in[1, \infty)^{N}$, i.e., if $f(\mathbf{z})=\sum_{\gamma \in \mathbb{N}_{0}^{N}} f_{\gamma} \mathbf{z}^{\gamma}$ then

$$
\|f(\mathbf{z})\|_{\mathbf{0}, r, \mathbf{s}}=\sum_{\gamma \in \mathbb{N}_{0}^{N}}\left|f_{\gamma}\right| r^{|\gamma|}
$$

Lemma 2 Let $f, g \in \mathscr{O}\left(D_{R}^{N}\right), \alpha, \beta \in \mathbb{N}^{N}$. Then for the modified Nagumo norm defined by (6) the following properties hold:

(i) $\|f(\mathbf{z}) \cdot g(\mathbf{z})\|_{\alpha+\beta, r, \mathbf{s}} \leq\|f(\mathbf{z})\|_{\alpha, r, \mathbf{s}}\|g(\mathbf{z})\|_{\beta, r, \mathbf{s}}$,

(ii) $\|f(\mathbf{z}) \cdot g(\mathbf{z})\|_{\alpha, r, \mathbf{s}} \leq\|f(\mathbf{z})\|_{\alpha, r, \mathbf{s}}\|g(\mathbf{z})\|_{\mathbf{0}, r, \mathbf{s}}$.

Before we can move on to the proof of these facts, let us introduce the following technical lemma:

Lemma 3 Let $p, q$ be positive natural numbers. Then

$$
\sum_{k=0}^{n}\left(\begin{array}{c}
k+p-1 \\
k
\end{array}\right)\left(\begin{array}{c}
n-k+q-1 \\
n-k
\end{array}\right)=\left(\begin{array}{c}
n+p+q-1 \\
n
\end{array}\right) \text { for any } n \in \mathbb{N}_{0} .
$$

Proof First let us note that for any $x \in \mathbb{R}$ satisfying $|x|<1$ it is true that

$$
\frac{1}{1-x}=\sum_{n=0}^{\infty} x^{n}
$$

After differentiating both sides of (8) $p-1$ times we receive

$$
\frac{(p-1) !}{(1-x)^{p}}=\sum_{n=0}^{\infty} \frac{(n+p-1) !}{n !} x^{n} \text {. }
$$

Hence,

$$
\begin{aligned}
\frac{1}{(1-x)^{p+q}} & =\left(\sum_{n=0}^{\infty} \frac{(n+p-1) !}{n !(p-1) !} x^{n}\right)\left(\sum_{n=0}^{\infty} \frac{(n+q-1) !}{n !(q-1) !} x^{n}\right) \\
& =\sum_{n=0}^{\infty} \sum_{k=0}^{n}\left(\begin{array}{c}
k+p-1 \\
k
\end{array}\right)\left(\begin{array}{c}
n-k+q-1 \\
n-k
\end{array}\right) x^{n} .
\end{aligned}
$$

On the other hand, we have

$$
\frac{(p+q-1) !}{(1-x)^{p+q}}=\sum_{n=0}^{\infty} \frac{(n+p+q-1) !}{n !} x^{n},
$$

which leads to the conclusion of this proof.

Let us now return to Lemma 2.

Proof of Lemma 2 Let us fix multi-indices $\alpha, \beta \in \mathbb{N}^{N}$. By the definitions of the Nagumo norms and $\Theta_{s}^{(a)}(x)$ we have

$$
f(\mathbf{z}) \ll \frac{\|f(\mathbf{z})\|_{\alpha, r, \mathbf{s}}}{r^{|\alpha|}} \prod_{i=1}^{N} \sum_{n=0}^{\infty}\left(\begin{array}{c}
n+\alpha_{i}-1 \\
n
\end{array}\right)^{s_{i}} \frac{z_{i}^{n}}{r^{n}}
$$


and

$$
g(\mathbf{z}) \ll \frac{\|g(\mathbf{z})\|_{\beta, r, \mathbf{s}}}{r^{|\beta|}} \prod_{i=1}^{N} \sum_{n=0}^{\infty}\left(\begin{array}{c}
n+\beta_{i}-1 \\
n
\end{array}\right) \frac{s_{i}}{r_{i}^{n}} .
$$

From these two facts it follows that

$$
f(\mathbf{z}) \cdot g(\mathbf{z}) \ll \frac{\|f(\mathbf{z})\|_{\alpha, r, \mathbf{s}}\|g(\mathbf{z})\|_{\beta, r, \mathbf{s}}}{r^{|\alpha|+|\beta|}} \prod_{i=1}^{N} \sum_{n=0}^{\infty} \sum_{k=0}^{n}\left(\begin{array}{c}
k+\alpha_{i}-1 \\
k
\end{array}\right)^{s_{i}}\left(\begin{array}{c}
n-k+\beta_{i}-1 \\
n-k
\end{array}\right)^{s_{i}} \frac{z_{i}^{n}}{r^{n}} .
$$

Using Lemma 3 and the fact that $a^{s}+b^{s} \leq(a+b)^{s}$ for any $a, b>0$ and $s \geq 1$, we conclude that

$$
f(\mathbf{z}) \cdot g(\mathbf{z}) \ll \frac{\|f(\mathbf{z})\|_{\alpha, r, \mathbf{s}}\|g(\mathbf{z})\|_{\beta, r, \mathbf{s}}}{r^{|\alpha|+|\beta|}} \prod_{i=1}^{N} \sum_{n=0}^{\infty}\left(\begin{array}{c}
n+\alpha_{i}+\beta_{i}-1 \\
k
\end{array}\right)^{s_{i}} \frac{z_{i}^{n}}{r^{n}},
$$

which finishes the proof of (i).

To show (ii), first we note that $g(\mathbf{z})=\sum_{\gamma \in \mathbb{N}_{0}^{N}} g_{\gamma} \mathbf{z}^{\gamma}$. Analogously to the proof of (i) we can write:

$$
\begin{aligned}
f(\mathbf{z}) \cdot g(\mathbf{z}) & \ll \frac{\|f(\mathbf{z})\|_{\alpha, r, \mathbf{s}}}{r^{|\alpha|}}\left(\prod_{i=1}^{N} \sum_{n=0}^{\infty}\left(\begin{array}{c}
n+\alpha_{i}-1 \\
n
\end{array}\right)^{s_{i}} \frac{z_{i}^{n}}{r^{n}}\right)\left(\sum_{\gamma \in \mathbb{N}_{0}^{N}}\left|g_{\gamma}\right| r^{|\gamma|} \frac{\mathbf{z}^{\gamma}}{r^{|\gamma|}}\right) \\
& =\frac{\|f(\mathbf{z})\|_{\alpha, r, \mathbf{s}}}{r^{|\alpha|}}\left(\sum_{\gamma \in \mathbb{N}_{0}^{N}} \prod_{i=1}^{N}\left(\begin{array}{c}
\gamma_{i}+\alpha_{i}-1 \\
\gamma_{i}
\end{array}\right)^{s_{i}} \frac{\mathbf{z}^{\gamma}}{r^{|\gamma|}}\right)\left(\sum_{\gamma \in \mathbb{N}_{0}^{N}}\left|g_{\gamma}\right| r^{|\gamma|} \frac{\mathbf{z}^{\gamma}}{r^{|\gamma|}}\right) \\
& =\frac{\|f(\mathbf{z})\|_{\alpha, r, \mathbf{s}}}{r^{|\alpha|}} \sum_{\gamma \in \mathbb{N}_{0}^{N}} \sum_{\gamma^{\prime} \leq \gamma} \prod_{i=1}^{N}\left(\begin{array}{c}
\gamma_{i}^{\prime}+\alpha_{i}-1 \\
\gamma_{i}^{\prime}
\end{array}\right)^{s_{i}}\left|g_{\gamma-\gamma^{\prime}}\right| r^{\left|\gamma-\gamma^{\prime}\right|} \frac{\mathbf{z}^{\gamma}}{r^{|\gamma|}} .
\end{aligned}
$$

Since for any $p \geq 0$ and $k \leq n$ we have $\left(\begin{array}{c}k+p \\ k\end{array}\right) \leq\left(\begin{array}{c}n+p \\ n\end{array}\right)$, it follows that

$$
\begin{aligned}
f(\mathbf{z}) \cdot g(\mathbf{z}) & \ll \frac{\|f(\mathbf{z})\|_{\alpha, r, \mathbf{s}}}{r^{|\alpha|}} \sum_{\gamma \in \mathbb{N}_{0}^{N}} \prod_{i=1}^{N}\left(\begin{array}{c}
\gamma_{i}+\alpha_{i}-1 \\
\gamma_{i}
\end{array}\right)^{s_{i}}\left(\sum_{\gamma^{\prime} \leq \gamma}\left|g_{\gamma-\gamma^{\prime}}\right| r^{\left|\gamma-\gamma^{\prime}\right|}\right) \frac{\mathbf{z}^{\gamma}}{r^{|\gamma|}} \\
& \ll \frac{\|f(\mathbf{z})\|_{\alpha, r, \mathbf{s}}\|g(\mathbf{z})\|_{\mathbf{0}, r, \mathbf{s}}}{r^{|\alpha|}} \sum_{\gamma \in \mathbb{N}_{0}^{N}} \prod_{i=1}^{N}\left(\begin{array}{c}
\gamma_{i}+\alpha_{i}-1 \\
\gamma_{i}
\end{array}\right) \frac{\mathbf{z}^{\gamma}}{r^{|\gamma|}} .
\end{aligned}
$$

Lemma 4 Assume that $\alpha \in \mathbb{N}^{N}$ and $m_{1}, \ldots, m_{N}$ are regular Gevrey-type sequences of orders $s_{1}, \ldots, s_{N}$, respectively, with every $s_{j} \geq 1$. Then

$$
\left\|\partial_{m_{j}, z_{j}} f(\mathbf{z})\right\|_{\alpha+\mathbf{e}_{j}, r, \mathbf{s}} \leq C \alpha_{j}^{s_{j}}\|f(\mathbf{z})\|_{\alpha, r, \mathbf{s}}
$$

for $j=1, \ldots, N$, where $\mathbf{e}_{j}$ denotes a multi-index with a 1 in the $j$ th coordinate and zeros everywhere else. 
Proof Since $m_{j}$ is a regular Gevrey-type sequence, there exist $c, C>0$ such that

$$
c(n+1)^{s_{j}} \leq \frac{m_{j}(n+1)}{m_{j}(n)} \leq C(n+1)^{s_{j}} \text { for any } n \in \mathbb{N}_{0} .
$$

Let us now fix $\alpha \in \mathbb{N}^{N}$. It follows that:

$$
\begin{aligned}
\partial_{m_{j}, z_{j}} f(\mathbf{z}) & \ll \frac{\|f(\mathbf{z})\|_{\alpha, r, \mathbf{s}}}{r^{\alpha_{j}}\left(\alpha_{j}-1\right) !^{s_{j}}} \sum_{n=0}^{\infty} \frac{\left(n+\alpha_{j}\right) !^{s_{j}} m_{j}(n+1)}{(n+1) !^{s_{j}} m_{j}(n)} \frac{z_{j}^{n}}{r^{n+1}} \prod_{i \neq j} \frac{1}{r^{\alpha_{i}}\left(\alpha_{i}-1\right) !^{s_{i}}} \Theta_{s_{i}}^{\left(\alpha_{i}-1\right)}\left(\frac{z_{i}}{r}\right) \\
& \ll \frac{\|f(\mathbf{z})\|_{\alpha, r, \mathbf{s}}}{r^{\alpha_{j}+1}\left(\alpha_{j}-1\right) !^{s_{j}}} \sum_{n=0}^{\infty} \frac{C\left(n+\alpha_{j}\right) !^{s_{j}}(n+1){ }^{s_{j}}}{(n+1) !^{s_{j}}} \frac{z_{j}^{n}}{r^{n}} \prod_{i \neq j} \frac{1}{r^{\alpha_{i}}\left(\alpha_{i}-1\right) !^{s_{i}}} \Theta_{s_{i}}^{\left(\alpha_{i}-1\right)}\left(\frac{z_{i}}{r}\right) \\
& =\frac{C\|f(\mathbf{z})\|_{\alpha, r, \mathbf{s}}}{r^{\alpha_{j}+1}\left(\alpha_{j}-1\right) !^{s_{j}}} \sum_{n=0}^{\infty} \frac{\left(n+\alpha_{j}\right) !^{s_{j}}}{n !^{s_{j}}} \frac{z_{j}^{n}}{r^{n}} \prod_{i \neq j} \frac{1}{r^{\alpha_{i}}\left(\alpha_{i}-1\right) !^{s_{i}}} \Theta_{s_{i}}^{\left(\alpha_{i}-1\right)}\left(\frac{z_{i}}{r}\right) \\
& =C \alpha_{j}^{s_{j}}\|f(\mathbf{z})\|_{\alpha, r, \mathbf{s}} \prod_{i=1}^{N} \frac{1}{r^{\alpha_{i}}\left(\tilde{\alpha_{i}}-1\right) !^{s_{i}}} \Theta_{s_{i}}^{\left(\tilde{\alpha}_{i}-1\right)}\left(\frac{z_{i}}{r}\right),
\end{aligned}
$$

where $\tilde{\alpha}=\alpha+\mathbf{e}_{j}$. From this it follows that $\left\|\partial_{m_{j}, z_{j}} f(\mathbf{z})\right\|_{\tilde{\alpha}, r, \mathbf{s}} \leq C \alpha_{j}^{s_{j}}\|f(\mathbf{z})\|_{\alpha, r, \mathbf{s}}$.

Lemma 5 Let $f \in \mathscr{O}\left(D_{R}^{N}\right)$. Then

$$
\|f(\mathbf{z})\|_{\alpha+\beta, r, \mathbf{s}} \leq r^{|\beta|}\|f(\mathbf{z})\|_{\alpha, r, \mathbf{s}}
$$

for any $0<r<R, \alpha \in \mathbb{N}^{N} \cup\{\mathbf{0}\}$ and $\beta \in \mathbb{N}^{N}$.

Proof This fact follows directly from Lemma 2. More precisely, it is true that $\|f(\mathbf{z})\|_{\alpha+\beta, r, \mathbf{s}} \leq\|1\|_{\beta, r, \mathbf{s}}\|f(\mathbf{z})\|_{\alpha, r, \mathbf{s}}$. Moreover, $\|1\|_{\beta, r, \mathbf{s}}=r^{|\beta|}$, because

$$
1 \ll \sum_{\gamma \in \mathbb{N}_{0}^{N}} \frac{z^{\gamma}}{r^{|\gamma|}}
$$

and the same is not true for $(1-\varepsilon) \sum_{\gamma \in \mathbb{N}_{0}^{N}} \frac{z^{\gamma}}{r^{|\gamma|}}$, no matter how small $\varepsilon>0$ we choose. Hence, $1=\frac{\|1\|_{\beta, r, s}}{r^{|\beta|}}$, which finishes the proof.

The following two lemmas connect Nagumo norms with the Gevrey order of formal power series.

Lemma 6 Let us consider $\mathbb{E}=\mathcal{O}\left(D_{R}^{N}\right) \cap C\left(\bar{D}_{R}^{N}\right)$ and $f(t, \mathbf{z})=\sum_{n=0}^{\infty} f_{n}(\mathbf{z}) t^{n} \in \mathbb{E}[[t]]_{w}$ with $w \geq 0$. Then for any $0<r<R$ and $\alpha \in \mathbb{N}^{N}$ there exist $A, B>0$ that satisfy

$$
\left\|f_{n}(\mathbf{z})\right\|_{n \alpha, r, \mathbf{s}} \leq A B^{n} n !^{w} \text { for any } n \in \mathbb{N}_{0} .
$$

Proof Since $f(t, \mathbf{z})$ is of Gevrey order $w$, for any $0<r<R$ there exist $\tilde{A}, \tilde{B}>0$ such that

$$
\sup _{\zeta \in D_{r}^{N}}\left|f_{n}(\zeta)\right| \leq \tilde{A} \tilde{B}^{n} n !^{w} \text { for } n \in \mathbb{N}_{0}
$$


Moreover, since $f_{n}(\mathbf{z}) \in \mathscr{O}\left(D_{R}^{N}\right)$, we can use Cauchy Inequalities for the power series of the form $f_{n}(\mathbf{z})=\sum_{\gamma \in \mathbb{N}_{0}^{N}} f_{n, \gamma} \mathbf{z}^{\gamma}$. More precisely, for any $0<r<R$ and $\gamma \in \mathbb{N}_{0}^{N}$ we have

$$
\left|f_{n, \gamma}\right| \leq \frac{\sup _{\zeta \in D_{r}^{N}}\left|f_{n}(\zeta)\right|}{r^{|\gamma|}}
$$

Hence,

$$
f_{n}(\mathbf{z}) \ll \sup _{\zeta \in D_{r}^{N}}\left|f_{n}(\zeta)\right| \sum_{\gamma \in \mathbb{N}_{0}^{N}} \frac{\mathbf{z}^{\gamma}}{r^{|\gamma|}} .
$$

First suppose that $n \geq 1$. Seeing as $\left(\begin{array}{c}m \\ l\end{array}\right) \geq 1$ for any $l \leq m$, we receive

$$
\begin{aligned}
f_{n}(\mathbf{z}) & \ll \sup _{\zeta \in D_{r}^{N}}\left|f_{n}(\zeta)\right| \prod_{i=1}^{N} \sum_{k=0}^{\infty}\left(\begin{array}{c}
k+n \alpha_{i}-1 \\
k
\end{array}\right)^{s_{i}} \frac{z_{i}^{k}}{r^{k}} \\
& \ll \sup _{\zeta \in D_{r}^{N}}\left|f_{n}(\zeta)\right| \prod_{i=1}^{N} \frac{R^{n \alpha_{i}}}{r^{n \alpha_{i}}} \sum_{k=0}^{\infty}\left(\begin{array}{c}
k+n \alpha_{i}-1 \\
k
\end{array}\right) \frac{s_{i}}{r^{k}} . \\
& \ll \sup _{\zeta \in D_{r}^{N}}\left|f_{n}(\zeta)\right| R^{n|\alpha|} \prod_{i=1}^{N} \frac{1}{r^{n \alpha_{i}}} \sum_{k=0}^{\infty}\left(\begin{array}{c}
k+n \alpha_{i}-1 \\
k
\end{array}\right)^{s_{i}} \frac{z_{i}^{k}}{r^{k}} .
\end{aligned}
$$

From this it follows that $\left\|f_{n}(\mathbf{z})\right\|_{n \alpha, r, \mathbf{z}} \leq R^{n|\alpha|} \sup _{\zeta \in D_{r}^{N}}\left|f_{n}(\zeta)\right| \leq \tilde{A}\left(\tilde{B} R^{|\alpha|}\right)^{n} n !^{w}$.

For $n=0$ let us notice that $f_{0}(\mathbf{z}) \in \mathscr{O}\left(D_{R}^{N}\right) \cap C\left(\bar{D}_{R}^{N}\right)$ and

$$
f_{0}(\mathbf{z})=\sum_{\gamma \in \mathbb{N}_{0}^{N}} f_{0, \gamma} \mathbf{z}^{\gamma}
$$

Then $\left|f_{0, \gamma}\right| \leq \frac{\sup _{\zeta \in D_{R}^{N}}\left|f_{0}(\zeta)\right|}{R^{|\gamma|}}$ for any $\gamma \in \mathbb{N}_{0}^{N}$ and

$$
\left\|f_{0}(z)\right\|_{\mathbf{0}, r, \mathbf{s}} \leq \sup _{\zeta \in D_{R}^{N}}\left|f_{0}(\zeta)\right| \sum_{\gamma \in \mathbb{N}_{0}^{N}} \frac{r^{k}}{R^{k}} \leq \sup _{\zeta \in D_{R}^{N}}\left|f_{0}(\zeta)\right| \frac{R^{N}}{(R-r)^{N}} .
$$

Hence it is enough to put $A=\max \left\{\tilde{A}, \sup _{\zeta \in D_{R}^{N}}\left|f_{0}(\zeta)\right| \frac{R^{N}}{(R-r)^{N}}\right\}$ and $B=\tilde{B} R^{|\alpha|}$.

Lemma 7 Let $f \in \mathscr{O}\left(D_{R}^{N}\right)$. Then for any $0<\rho<r<R$ there exists $A>0$ such that for any $\alpha \in \mathbb{N}^{N} \cup\{\mathbf{0}\}$ the following inequality holds:

$$
\sup _{\mathbf{z} \in D_{\rho}^{N}}|f(\mathbf{z})| \leq A^{|\alpha|}\|f(\mathbf{z})\|_{\alpha, r, \mathbf{s}} .
$$

Proof When $\alpha=\mathbf{0}$ it suffices to notice that for certain $\theta_{1}, \ldots, \theta_{N} \in[0,2 \pi)$ we have

$$
\sup _{\mathbf{z} \in D_{\rho}^{N}}|f(\mathbf{z})|=\left|\sum_{\gamma \in \mathbb{N}_{0}^{N}} f_{\gamma} \prod_{i=1}^{N} \rho^{\gamma_{i}} e^{i \theta_{i} \gamma_{i}}\right| \leq \sum_{\gamma \in \mathbb{N}_{0}^{N}}\left|f_{\gamma}\right| \rho^{|\gamma|} \leq \sum_{\gamma \in \mathbb{N}_{0}^{N}}\left|f_{\gamma}\right| r^{\gamma}=\|f(\mathbf{z})\|_{\mathbf{0}, r, \mathbf{s}} .
$$


To prove the same for $\alpha \in \mathbb{N}^{N}$ first let us note that for any $a, b \geq 0, p \in \mathbb{N}$ and $n \in \mathbb{N}_{0}$ we have

$$
\left(\begin{array}{c}
n+p-1 \\
n
\end{array}\right) a^{n} b^{p-1} \leq(a+b)^{n+p-1} .
$$

In particular, if we set $\varepsilon>0$ small enough that $\rho(1+\varepsilon)^{|\mathbf{s}|-N}<r$ then (9) holds for $a+b=1$ and $a^{-1}=1+\varepsilon$. Then for any $n \in \mathbb{N}_{0}$ and $i=1, \ldots, N$ we get

$$
\left(\begin{array}{c}
n+\alpha_{i}-1 \\
n
\end{array}\right) \leq\left(\frac{1+\varepsilon}{\varepsilon}\right)^{\alpha_{i}-1}(1+\varepsilon)^{n} .
$$

Now we can apply this fact to majorize $f(\mathbf{z})$ in the following way:

$$
\begin{aligned}
f(\mathbf{z}) & \ll \frac{\|f(\mathbf{z})\|_{\alpha, r, \mathbf{s}}}{r^{|\alpha|}} \prod_{i=1}^{N} \sum_{n=0}^{\infty}\left(\begin{array}{c}
n+\alpha_{i}-1 \\
n
\end{array}\right)^{s_{i}} \frac{z_{i}^{n}}{r^{n}} \\
& \ll \frac{\|f(\mathbf{z})\|_{\alpha, r, \mathbf{s}}}{r^{|\alpha|}} \prod_{i=1}^{N} \sum_{n=0}^{\infty}\left(\frac{1+\varepsilon}{\varepsilon}\right)^{\left(\alpha_{i}-1\right)\left(s_{i}-1\right)}(1+\varepsilon)^{n\left(s_{i}-1\right)}\left(\begin{array}{c}
n+\alpha_{i}-1 \\
n
\end{array}\right) \frac{z_{i}^{n}}{r^{n}} \\
& \ll \frac{\|f(\mathbf{z})\|_{\alpha, r, \mathbf{s}}}{r^{|\alpha|}}\left(\frac{1+\varepsilon}{\varepsilon}\right)^{\mathbf{s} \cdot \alpha} \prod_{i=1}^{N} \sum_{n=0}^{\infty}\left(\begin{array}{c}
n+\alpha_{i}-1 \\
n
\end{array}\right)\left(\frac{z_{i}(1+\varepsilon)^{s_{i}-1}}{r}\right)^{n} .
\end{aligned}
$$

In particular, the majorization above is true for any $\mathbf{z} \in D_{\rho}^{N}$ and so we can conclude that

$$
\sup _{\mathbf{z} \in D_{\rho}^{N}}|f(\mathbf{z})| \leq \frac{\|f(\mathbf{z})\|_{\alpha, r, \mathbf{s}}}{r^{|\alpha|}}\left(\frac{1+\varepsilon}{\varepsilon}\right)^{\mathbf{s} \cdot \alpha} \prod_{i=1}^{N} \sum_{n=0}^{\infty}\left(\begin{array}{c}
n+\alpha_{i}-1 \\
n
\end{array}\right)\left(\frac{\rho(1+\varepsilon)^{s_{i}-1}}{r}\right)^{n} .
$$

Moreover, because of the properties of our chosen $\varepsilon$, for $i=1, \ldots, N$ we have

$$
\sum_{n=0}^{\infty} \frac{\left(n+\alpha_{i}-1\right) !}{n !}\left(\frac{\rho(1+\varepsilon)^{s_{i}-1}}{r}\right)^{n}=\left.\frac{d^{\alpha_{i}-1}}{d u^{\alpha_{i}-1}} \frac{1}{1-u}\right|_{u=\rho(1+\varepsilon)^{s_{i}-1} r^{-1}} .
$$

From this fact it follows that

$$
\begin{aligned}
\sup _{\mathbf{z} \in D_{\rho}^{N}}|f(\mathbf{z})| & \leq \frac{\|f(\mathbf{z})\|_{\alpha, r, \mathbf{s}}}{r^{|\alpha|}}\left(\frac{1+\varepsilon}{\varepsilon}\right)^{\mathbf{s} \cdot \alpha} \frac{r^{|\alpha|}}{\left[r-(1+\varepsilon)^{|\mathbf{s}|-N} \rho\right]^{|\alpha|}} \\
& \leq\|f(\mathbf{z})\|_{\alpha, r, \mathbf{s}}\left\{\frac{(1+\varepsilon)^{|\mathbf{s}|}}{\varepsilon^{|\mathbf{s}|}\left[r-(1+\varepsilon)^{|\mathbf{s}|-N} \rho\right]}\right\}^{|\alpha|} .
\end{aligned}
$$

Therefore it is enough to put $A=\max \left\{1, \frac{(1+\varepsilon)^{|\mathbf{s}|}}{\varepsilon^{|\mathbf{s}|}\left[r-(1+\varepsilon)^{|\mathbf{s}|-N} \rho\right]}\right\}$.

\section{The Linear Cauchy Problem}

Suppose that $t \in \mathbb{C}$ and $\mathbf{z} \in \mathbb{C}^{N}, N \geq 1$, and let $\mathbf{m}=\left(m_{1}, \ldots, m_{N}\right)$, where $m_{0}, m_{1}, \ldots, m_{N}$ are regular Gevrey-type sequences of non-negative orders $s_{0}, s_{1}, \ldots, s_{N}$, respectively. Then we define the Gevrey-type differential operator $P\left(\partial_{m_{0}, t}, \partial_{\mathbf{m}, \mathbf{z}}\right)$ by the formula:

$$
P\left(\partial_{m_{0}, t}, \partial_{\mathbf{m}, \mathbf{z}}\right)=\partial_{m_{0}, t}^{M}+\sum_{(j, \alpha) \in \Lambda} a_{j, \alpha}(t, \mathbf{z}) \partial_{m_{0}, t}^{j} \partial_{\mathbf{m}, \mathbf{z}}^{\alpha}
$$

with additional assumptions: 
(a) $\mathbf{s}=\left(s_{1}, s_{2}, \ldots, s_{N}\right) \in[1, \infty)^{N}$,

(b) $\Lambda \subset \mathbb{N}_{0}^{1+N}$ is a finite set of indices,

(c) $\operatorname{ord}_{t}\left(a_{j, \alpha}\right) \geq \max \{0, j-M+1\}$ for all $(j, \alpha) \in \Lambda$.

Our first step is to analyze the Newton polygon for (10). In this case it is given by

$$
N\left(P, s_{0}, \mathbf{s}\right)=\mathrm{conv}\left\{\Delta\left(s_{0} M,-M\right) \cup \bigcup_{(j, \alpha) \in \Lambda} \Delta\left(s_{0} j+\mathbf{s} \cdot \alpha, \operatorname{ord}_{t}\left(a_{j, \alpha}\right)-j\right)\right\} .
$$

Note that the first non-horizontal segment (if it exists) of the boundary of $N\left(P, s_{0}, \mathbf{s}\right)$ connects points with coordinates $\left(s_{0} M,-M\right)$ and $\left(s_{0} j^{*}+\mathbf{s} \cdot \alpha^{*}, \operatorname{ord}_{t}\left(a_{j^{*}, \alpha^{*}}\right)-j^{*}\right)$ for a certain $\left(j^{*}, \alpha^{*}\right) \in \Lambda$. The slope of this segment is positive and given by the formula

$$
k_{1}=\frac{\operatorname{ord}_{t}\left(a_{j^{*}, \alpha^{*}}\right)-j^{*}+M}{s_{0}\left(j^{*}-M\right)+\mathbf{s} \cdot \alpha^{*}} .
$$

Alternatively, we can define $k_{1}$ using the formula

$$
\frac{1}{k_{1}}=\max \left\{0, \max _{(j, \alpha) \in \Lambda}\left\{\frac{s_{0}(j-M)+\mathbf{s} \cdot \alpha}{\operatorname{ord}_{t}\left(a_{j, \alpha}\right)-j+M}\right\}\right\} .
$$

Let us consider a linear Cauchy problem of the form

$$
\left\{\begin{array}{rl}
P\left(\partial_{m_{0}, t}, \partial_{\mathbf{m}, \mathbf{z}}\right) u(t, \mathbf{z}) & =f(t, \mathbf{z}) \\
\partial_{m_{0}, t}^{j} u(0, \mathbf{z}) & =\phi_{j}(\mathbf{z}) \text { for } 0 \leq j<M
\end{array},\right.
$$

further assuming that:

(d) $f(t, \mathbf{z}) \in \mathscr{O}\left(D_{R}^{N}\right)[[t]]_{1 / k_{1}}$ and $a_{j, \alpha}(t, \mathbf{z}) \in \mathscr{O}\left(D_{R}^{N}\right)[[t]]_{1 / k_{1}}$ for all $(j, \alpha) \in \Lambda$,

(e) $\phi_{j} \in \mathscr{O}\left(D_{R}^{N}\right)$ for $0 \leq j<M$.

Our aim is to prove the following theorem:

Theorem 1 Under assumptions (a)-(e) listed above the formal solution $\hat{u}(t, \mathbf{z})=$ $\sum_{n=0}^{\infty} u_{n}(\mathbf{z}) t^{n}$ of (11) is of the Gevrey order $\frac{1}{k_{1}}$.

Before moving on to the proof of this theorem, we shall show that the following proposition holds true:

Proposition 2 Suppose that $\alpha_{0}=\left(\alpha_{0,1}, \alpha_{0,2}, \ldots, \alpha_{0, N}\right)$ is a multi-index such that

$$
\alpha_{0, k}:=\left\lfloor\max _{(j, \alpha) \in \Lambda} \frac{\alpha_{k}}{\operatorname{ord}_{t}\left(a_{j, \alpha}\right)-j+M}\right\rfloor+1 \text { for } k=1,2, \ldots, N
$$

and that $\hat{u}(t, \mathbf{z})=\sum_{n=0}^{\infty} u_{n}(\mathbf{z}) t^{n}$ is a formal solution of (11). Then for any $0<r<R$ there exist constants $A, B>0$ such that

$$
\left\|u_{n}(z)\right\|_{n \alpha_{0}, r, \mathbf{s}} \leq A B^{n} n !^{1 / k_{1}} \text { for any } n \in \mathbb{N}_{0} .
$$

Proof Without any loss of generality we may assume that $u_{0}(\mathbf{z}) \equiv 0$. Otherwise it is enough to use substitution $v(t, \mathbf{z}):=u(t, \mathbf{z})-\phi_{0}(\mathbf{z})$.

Now we can proceed to show (12) by induction on $n$. It is easy to see that (12) holds for $n \leq M-1$. This fact follows directly from the properties of functions $\phi_{1}(\mathbf{z}), \ldots, \phi_{M-1}(\mathbf{z})$ 
and Lemma 6. Let us then show that if $n \geq M$ and the proposition (with $n$ replaced by $i$ ) holds for any $i<n$ then it is also true for $n$.

Let $t^{M} f(t, \mathbf{z})=\sum_{n=M}^{\infty} f_{n}(\mathbf{z}) t^{n}$ and $t^{M-j} a_{j, \alpha}(t, \mathbf{z})=\sum_{n=q_{j, \alpha}}^{\infty} a_{j, \alpha, n}(\mathbf{z}) t^{n}$ with $q_{j, \alpha}=$ $\operatorname{ord}_{t}\left(a_{j, \alpha}(t, \mathbf{z})\right)-j+M$ for every $(j, \alpha) \in \Lambda$. Using these facts we find a recurrence relation describing $u_{n}(\mathbf{z})$ for $n \geq M$ :

$$
u_{n}(\mathbf{z})=\frac{m_{0}(n-M)}{m_{0}(n)}\left[f_{n}(\mathbf{z})-\sum_{(j, \alpha) \in \Lambda} \sum_{p=q_{j, \alpha}}^{n} a_{j, \alpha, p}(\mathbf{z}) \frac{m_{0}(n-p)}{m_{0}(n-p-j)} \partial_{\mathbf{m}, \mathbf{z}}^{\alpha} u_{n-p}(\mathbf{z})\right] .
$$

From this point on we shall use a convention that a term $\frac{m_{0}(n-p)}{m_{0}(n-p-j)}$ disappears whenever $n-p-j<0$.

Let us fix $0<r<R$. By assumption (d) and Lemma 6 there exist positive constants $F, G$, for which $\left\|f_{n}(\mathbf{z})\right\|_{n \alpha_{0}, r, \mathbf{s}} \leq F G^{n} n !^{1 / k_{1}}$. Moreover, from regularity of $m_{0}$ and $m_{1}, \ldots, m_{N}$, there exist constants $c, C>0$ such that

$$
c(n+1)^{s_{j}} \leq \frac{m_{j}(n+1)}{m_{j}(n)} \leq C(n+1)^{s_{j}} \text { for any } n \in \mathbb{N}_{0} \text { and } j=0,1, \ldots, N .
$$

Hence, we have

$$
\begin{aligned}
\left\|u_{n}(\mathbf{z})\right\|_{n \alpha_{0}, r, \mathbf{s}} \leq \frac{c^{-M}(n-M) !^{s_{0}}}{n !^{s_{0}}}\left[F G^{n} n !^{1 / k_{1}}\right. & +\sum_{(j, \alpha) \in \Lambda} \sum_{p=q_{j, \alpha}}^{n} C^{j} \frac{(n-p) !^{s_{0}}}{(n-p-j) !^{s_{0}}} \\
& \left.\times\left\|a_{j, \alpha, p}(\mathbf{z}) \partial_{\mathbf{m}, \mathbf{z}}^{\alpha} u_{n-p}(\mathbf{z})\right\|_{n \alpha_{0}, r, \mathbf{s}}\right] .
\end{aligned}
$$

It is easy to see that the first term on the right-hand side of the inequality above can be bounded by $\frac{1}{2} A B^{n} n !^{1 / k_{1}}$ if we put $A \geq 2 c^{-M} F$ and $B \geq G$.

Now we can analyze the term:

$$
I=\frac{c^{-M}(n-M) !^{s_{0}}}{n !^{s_{0}}} \sum_{(j, \alpha) \in \Lambda} \sum_{p=q_{j, \alpha}}^{n} C^{j} \frac{(n-p) !^{s_{0}}}{(n-p-j) !^{s_{0}}}\left\|a_{j, \alpha, p}(\mathbf{z}) \partial_{\mathbf{m}, \mathbf{z}}^{\alpha} u_{n-p}(\mathbf{z})\right\|_{n \alpha_{0}, r, \mathbf{s}},
$$

By Lemma 2 we have

$$
\left\|a_{j, \alpha, p}(\mathbf{z}) \partial_{\mathbf{m}, \mathbf{z}}^{\alpha} u_{n-p}(\mathbf{z})\right\|_{n \alpha_{0}, r, \mathbf{s}} \leq\left\|a_{j, \alpha, p}(\mathbf{z})\right\|_{p \alpha_{0}-\alpha, r, \mathbf{s}}\left\|\partial_{\mathbf{m}, \mathbf{z}}^{\alpha} u_{n-p}(\mathbf{z})\right\|_{(n-p) \alpha_{0}+\alpha, r, \mathbf{s}} .
$$

By Lemma 5 and the definition of $\alpha_{0}$ we also get

$$
\left\|a_{j, \alpha, p}(\mathbf{z})\right\|_{p \alpha_{0}-\alpha, r, \mathbf{s}} \leq R^{q_{j, \alpha}\left|\alpha_{0}\right|-|\alpha|}\left\|a_{j, \alpha, p}(\mathbf{z})\right\|_{\left(p-q_{j, \alpha}\right) \alpha_{0}, r, \mathbf{s}} .
$$

Note that $q_{j, \alpha} \geq 1$ for every $(j, \alpha) \in \Lambda$ and so we can write that $t^{M-j} a_{j, \alpha}(t, \mathbf{z})=$ $t^{q_{j, \alpha}} \sum_{n=q_{j, \alpha}}^{\infty} a_{j, \alpha, n}(\mathbf{z}) t^{n-q_{j, \alpha}}$ and treat every $a_{j, \alpha, n}(\mathbf{z})$ like the $\left(n-q_{j, \alpha}\right)$ th coefficient of the formal power series. Hence, using Lemma 6 we receive

$$
\left\|a_{j, \alpha, p}(\mathbf{z})\right\|_{\left(p-q_{j, \alpha}\right) \alpha_{0}, r, \mathbf{s}} \leq \tilde{K} L^{p}\left(p-q_{j, \alpha}\right) !^{1 / k_{1}}
$$

for a certain pair of positive constants $\tilde{K}, L$. If we put $K=R^{q_{j, \alpha}\left|\alpha_{0}\right|} \tilde{K}$ then

$$
\left\|a_{j, \alpha, p}(\mathbf{z})\right\|_{p \alpha_{0}-\alpha, r, \mathbf{s}} \leq K L^{p}\left(p-q_{j, \alpha}\right) !^{1 / k_{1}} .
$$


Moreover, by Lemma 4 we have

$$
\begin{aligned}
\left\|\partial_{\mathbf{m}, \mathbf{z}}^{\alpha} u_{n-p}(\mathbf{z})\right\|_{(n-p) \alpha_{0}+\alpha, r, \mathbf{s}} \leq & C^{|\alpha|}\left|\alpha_{0}\right|^{\mathbf{s} \cdot \alpha}\left\|u_{n-p}(\mathbf{z})\right\|_{(n-p) \alpha_{0}, r, \mathbf{s}} \\
& \times \prod_{k=1}^{N}\left(n-p+\frac{\alpha_{k}-1}{\alpha_{0, k}}\right)^{s_{k}}\left(n-p+\frac{\alpha_{k}-2}{\alpha_{0, k}}\right)^{s_{k}} \\
& \ldots(n-p)^{s_{k}}
\end{aligned}
$$

and, by the inductive assumption,

$$
\left\|u_{n-p}(\mathbf{z})\right\|_{(n-p) \alpha_{0}, r, \mathbf{s}} \leq A B^{n-p}(n-p) !^{1 / k_{1}} .
$$

Furthermore, let us observe that

$$
\left(p-q_{j, \alpha}\right) !^{1 / k_{1}}(n-p) !^{1 / k_{1}} \leq \frac{n !^{1 / k_{1}}}{(n-p+1)^{1 / k_{1}} \ldots\left(n-p+q_{j, \alpha}\right)^{1 / k_{1}}} \leq \frac{n !^{1 / k_{1}}}{(n-p+1)^{q_{j, \alpha} / k_{1}}}
$$

and

$$
n-p+\frac{l}{\alpha_{0, k}} \leq n-p+\frac{q_{j, \alpha} l}{\alpha_{k}} \text { for any } 0 \leq l \leq \alpha_{k}-1,1 \leq k \leq N .
$$

Hence,

$$
\begin{aligned}
& I \leq A B^{n} n !^{1 / k_{1}} \sum_{(j, \alpha) \in \lambda} c^{-M} K C^{j+|\alpha|}\left|\alpha_{0}\right|^{\mathbf{s} \cdot \alpha} \frac{(n-M) !^{s_{0}}(n-p) !^{s_{0}}}{n !^{s_{0}}(n-p-j) !^{!_{0}}(n-p+1)^{q_{j, \alpha} / k_{1}}} \\
& \times \prod_{k=1}^{N} \prod_{l=0}^{\alpha_{k}-1}\left(n-p+\left\lceil\frac{q_{j, \alpha} l}{\alpha_{k}}\right\rceil\right)^{s_{k}} \sum_{p=q_{j, \alpha}}^{n}\left(\frac{L}{B}\right)^{p} \\
& \leq A B^{n} n !^{1 / k_{1}} \sum_{(j, \alpha) \in \lambda} c^{-M} K C^{j+|\alpha|}\left|\alpha_{0}\right|^{\mathbf{s} \cdot \alpha} \frac{(n-p)^{s_{0} j}\left(n-p+q_{j, \alpha}\right)^{\mathbf{s} \cdot \alpha}}{(n-M+1)^{s_{0} M}(n-p+1)^{s_{0}(j-M)+\mathbf{s} \cdot \alpha}} \\
& \times \sum_{p=q_{j, \alpha}}^{n}\left(\frac{L}{B}\right)^{p} \\
& \leq A B^{n} n !^{1 / k_{1}} \sum_{(j, \alpha) \in \lambda} c^{-M} K C^{j+|\alpha|}\left|\alpha_{0}\right|^{\mathbf{s} \cdot \alpha}\left(\frac{n-p+q_{j, \alpha}}{n-p+1}\right)^{\mathbf{s} \cdot \alpha}\left(\frac{n-p+1}{n-M+1}\right)^{s_{0} M} \\
& \times \sum_{p=q_{j, \alpha}}^{n}\left(\frac{L}{B}\right)^{p} .
\end{aligned}
$$

Moreover,

$$
\frac{n-p+q_{j, \alpha}}{n-p+1} \leq 1+\frac{q_{j, \alpha}-1}{n-p+1} \leq q_{j, \alpha} \text { for any } 1 \leq p \leq n
$$

and

$$
\frac{n-p+1}{n-M+1} \leq \frac{1}{1-\frac{M-1}{n}} \leq M \text { for any } n \geq M,
$$


By combining facts listed above we receive

$$
\begin{aligned}
I & \leq A B^{n} n !^{1 / k_{1}} \sum_{(j, \alpha) \in \Lambda} c^{-M} K C^{j+|\alpha|}\left|\alpha_{0}\right|^{\mathbf{s} \cdot \alpha} M^{M s_{0}} q_{j, \alpha}^{\mathbf{s} \cdot \alpha} \sum_{p=q_{j, \alpha}}^{n}\left(\frac{L}{B}\right)^{p} \\
& \leq A B^{n} n !^{1 / k_{1}} \sum_{(j, \alpha) \in \Lambda} c^{-M} K C^{j+|\alpha|}\left|\alpha_{0}\right|^{\mathbf{s} \cdot \alpha} M^{M s_{0}} q_{j, \alpha}^{\mathbf{s} \cdot \alpha} \sum_{p=q_{j, \alpha}}^{\infty}\left(\frac{L}{B}\right)^{p} \\
& =A B^{n} n !^{1 / k_{1}} \sum_{(j, \alpha) \in \Lambda} c^{-M} K C^{j+|\alpha|}\left|\alpha_{0}\right|^{\mathbf{s} \cdot \alpha} M^{M s_{0}} q_{j, \alpha}^{\mathbf{s} \cdot \alpha} \frac{L}{B-L} .
\end{aligned}
$$

It remains to notice that the last sum can be bounded from above by $\frac{1}{2}$ for sufficiently large $B>L$.

Now we can go back to the proof of Theorem 1.

Proof of Theorem 1 Using Proposition 2 and Lemma 7 we conclude that for any $n \in \mathbb{N}_{0}$ and $\rho<r$ there exist constants $\tilde{A}, A$, and $B$ such that

$$
\sup _{\mathbf{z} \in D_{\rho}^{N}}\left|u_{n}(\mathbf{z})\right| \leq \tilde{A}^{n\left|\alpha_{0}\right|}\left\|u_{n}(z)\right\|_{n \alpha_{0}, r, \mathbf{s}} \leq A\left(\tilde{A}^{\left|\alpha_{0}\right|} B\right)^{n} n !^{1 / k_{1}} .
$$

Hence, $\hat{u}(t, \mathbf{z}) \in \mathscr{O}\left(D_{R}^{N}\right)[[t]]_{1 / k_{1}}$.

\section{Suggestions for Further Research}

It might be possible to extend the result from Theorem 1 to a certain class of non-linear Gevrey-type differential equations. We would like to achieve this by using methods inspired by the approach presented in this paper.

Moreover, it is worth mentioning that an idea similar to the Gevrey-type sequences has already been considered in [4]. Sequences and operators defined there are similar to the ones presented in Definitions 2 and 3, with the Gevrey sequence $n !^{s}$ replaced with any given sequence $M_{n}$ of positive real numbers. Under certain additional assumptions concerning the sequence $M_{n}$ the authors were able to analyze the Gevrey properties of a certain class of linear equations with time-dependent coefficients using the approach similar to [10] and [7]. A question remains open, whether those findings can be extended to the case when the coefficients depend on both $t \in \mathbb{C}$ and $\mathbf{z} \in \mathbb{C}^{N}$.

Funding Information This study was supported by Cardinal Stefan Wyszynski University in Warsaw.

Open Access This article is licensed under a Creative Commons Attribution 4.0 International License, which permits use, sharing, adaptation, distribution and reproduction in any medium or format, as long as you give appropriate credit to the original author(s) and the source, provide a link to the Creative Commons licence, and indicate if changes were made. The images or other third party material in this article are included in the article's Creative Commons licence, unless indicated otherwise in a credit line to the material. If material is not included in the article's Creative Commons licence and your intended use is not permitted by statutory regulation or exceeds the permitted use, you will need to obtain permission directly from the copyright holder. To view a copy of this licence, visit http://creativecommonshorg/licenses/by/4.0/. 


\section{References}

1. Balser W. Formal power series and linear systems of meromorphic ordinary differential equations. New York: Springer; 2000.

2. Balser W, Loday-Richaud M. Summability of solutions of the heat equation with inhomogeneous thermal conductivity in two variables. Advances in Dynamical Systems and Applications. 2009;4(2):159-177.

3. Balser W, Yoshino M. Gevrey order of formal power series solutions of inhomogeneous partial differential equations with constant coefficients. Funkcial Ekvac. 2010;53:411-434.

4. Lastra M, Michalik S, Suwińska M. Estimates of formal solutions for some generalized moment partial differential equations. arXiv:1911.11998. 2020.

5. Michalik S. Analytic solutions of moment partial differential equations with constant coefficients. Funkcial Ekvac. 2013;56:19-50.

6. Michalik S. Analytic and summable solutions of inhomogeneous moment partial differential equations. Funkcial Ekvac. 2017;60:325-351.

7. Michalik S, Suwińska M. Gevrey estimates for certain moment partial differential equations. In: Complex Differential and Difference Equations, pp. 391-408. De Gruyter Proceedings in Mathematics; 2020.

8. Remy P. Gevrey order and summability of formal series solutions of some classes of inhomogeneous linear partial differential equations with variable coefficients. J Dyn Control Syst. 2016;22:693-711.

9. Remy P. Gevrey order and summability of formal series solutions of certain classes of inhomogeneous linear integro-differential equations with variable coefficients. J Dyn Control Syst. 2017;23:853-878.

10. Tahara H, Yamazawa H. Multisummability of formal solutions to the cauchy problem for some linear partial differential equations. J Differential Equations. 2013;255:3592-3637.

11. Yonemura A. Newton polygons and formal Gevrey classes. Publ RIMS Kyoto Univ. 1990;26:197-204.

Publisher's Note Springer Nature remains neutral with regard to jurisdictional claims in published maps and institutional affiliations. 